\title{
Genome wide analysis of DNA copy number neutral loss of heterozygosity (CNNLOH) and its relation to gene expression in esophageal squamous cell carcinoma
}

Nan Hu ${ }^{1 \dagger}$, Robert J Clifford ${ }^{2 \dagger}$, Howard H Yang ${ }^{2}$, Chaoyu Wang ${ }^{1}$, Alisa M Goldstein ${ }^{1}$, Ti Ding ${ }^{3}$, Philip R Taylor ${ }^{1 *}$, Maxwell P Lee 2* $^{*}$

\begin{abstract}
Background: Genomic instability plays an important role in human cancers. We previously characterized genomic instability in esophageal squamous cell carcinomas (ESCC) in terms of loss of heterozygosity (LOH) and copy number (CN) changes in tumors using the Affymetrix GeneChip Human Mapping 500K array in 30 cases from a high-risk region of China. In the current study we focused on copy number neutral (CN =2) LOH (CNNLOH) and its relation to gene expression in ESCC.

Results: Overall we found that $70 \%$ of all LOH observed was CNNLOH. Ninety percent of ESCCs showed CNNLOH (median frequency in cases $=60 \%$ ) and this was the most common type of $\mathrm{LOH}$ in two-thirds of cases. CNNLOH occurred on all 39 autosomal chromosome arms, with highest frequencies on 19p (100\%), 5p (96\%), 2p (95\%), and 20q (95\%). In contrast, LOH with CN loss represented 19\% of all LOH, occurred in just half of ESCCs (median frequency in cases $=0 \%$, and was most frequent on 3p (56\%), $5 q(47 \%)$, and $21 q(41 \%)$. LOH with CN gain was $11 \%$ of all LOH, occurred in $93 \%$ of ESCCs (median frequency in cases $=13 \%$ ), and was most common on 20p (82\%), $8 \mathrm{q}(74 \%)$, and $3 \mathrm{q}(42 \%)$. To examine the effect of genomic instability on gene expression, we evaluated RNA profiles from 17 pairs of matched normal and tumor samples (a subset of the 30 ESCCs) using Affymetrix U133A 2.0 arrays. In CN neutral regions, expression of 168 genes (containing 1976 SNPs) differed significantly in tumors with $\mathrm{LOH}$ versus tumors without $\mathrm{LOH}$, including 101 genes that were up-regulated and 67 that were downregulated.
\end{abstract}

Conclusion: Our results indicate that CNNLOH has a profound impact on gene expression in ESCC, which in turn may affect tumor development.

\section{Background}

Genomic instability is important for cancer development and can manifest as copy number $(\mathrm{CN})$ gain or loss as well as loss of heterozygosity (LOH). Copy number neutral $\mathrm{LOH}(\mathrm{CNNLOH})$ has been observed in tumors following the widespread application of SNP array

\footnotetext{
* Correspondence: ptaylor@mail.nih.gov; leemax@mail.nih.gov

+ Contributed equally

${ }^{1}$ Genetic Epidemiology Branch, Division of Cancer Epidemiology and Genetics, National Cancer Institute, NIH, DHHS, Bethesda, Maryland, USA ${ }^{2}$ Laboratory of Population Genetics, Center for Cancer Research, National Cancer Institute, NIH, DHHS, Bethesda, Maryland, USA

Full list of author information is available at the end of the article
}

technology $[1,2]$. CNNLOH is common in many tumor types, including basal cell carcinoma [3], acute myeloid leukemia [4,5], medulloblastoma [6], melanoma [7], follicular lymphoma [8], colorectal cancers [9-11], glioblastoma [12,13], cutaneous squamous cell carcinomas [14], acute promyelocytic leukemia [15], acute lymphoblastic leukemia [16], ovarian tumor [17], and esophageal adenocarcinoma [18], and has recently been reviewed for myeloid malignancies [19]. CNNLOH is thought to result from mitotic recombination or nondisjunction in somatic tumor cells [3]. However, the distribution of complex DNA alterations and its relation to gene 
expression in tumors have not been characterized in ESCC.

ESCC is a common malignancy worldwide and one of the most common cancers in the Chinese population; Shanxi Province in north central China has some of the highest esophageal cancer rates in the world $[20,21]$. Previously, we identified several regions of $\mathrm{LOH}$ and $\mathrm{CN}$ alteration in ESCC using microsatellite markers and lowand high-density SNP arrays [22-27], where the majority of ESCC patients from this high-risk population were found to have high genomic instability and high frequency of $\mathrm{LOH}$ on several chromosome arms. However, we have not found causal mutations in candidate genes within the LOH regions identified. For example, $82 \%$ of 56 ESCCs showed $\mathrm{LOH}$ when tested with four microsatellite markers flanking ANXA1 (9q11-q21), but no somatic mutations were detected in these patients [28]. Another example is $B R C A 2$, which also showed frequent $\mathrm{LOH}$ in ESCC (57\% for D13S260, 83\% for D13S767), but only infrequent somatic mutations in these cancer patients $(2 / 56,3.5 \%)$ $[29,30]$. Contrary to expectation, expression of $B R C A 2$ was often increased (unpublished data).

In the present study, we analyzed DNA from 30 microdissected ESCC tumors, adjacent normal tissue, and blood DNA from the same patient using the Affymetrix 500K SNP array to identify the distribution of complex DNA alterations, including $\mathrm{CNNLOH}$, and we related $\mathrm{CNNLOH}$ to expression of the genes affected as assessed with the Affymetrix U133A 2.0 array in these patients.

\section{Methods \\ Case selection}

This study was approved by the Institutional Review Boards of the Shanxi Cancer Hospital and the US National Cancer Institute (NCI). Cases diagnosed with ESCC between 1998 and 2001 in the Shanxi Cancer Hospital in Taiyuan, Shanxi Province, PR China, and considered candidates for curative surgical resection were identified and recruited to participate in this study. None of the cases had prior therapy and Shanxi was the ancestral home for all. After obtaining informed consent, cases were interviewed to obtain information on demographics, cancer risk factors (eg, smoking, alcohol drinking, and detailed family history of cancer), and clinical information. The cases evaluated here were part of a larger case-control study of upper gastrointestinal cancers conducted in Shanxi Province [31-33].

\section{Biological specimen collection and processing}

Venous blood $(10 \mathrm{ml})$ was taken from each case prior to surgery and germ-line DNA from whole blood was extracted and purified using the standard phenol/chloroform method.
Tumor and adjacent normal tissues were dissected at the time of surgery and stored in liquid nitrogen until used. One 5-micron section was H\&E stained and reviewed by a pathologist from the NCI to guide the micro-dissection. Five to ten consecutive 8-micron sections were cut from fresh frozen tumor and adjacent normal tissues. Tumor and normal cells were manually micro-dissected under light microscopy. DNA was extracted from micro-dissected tumor as previously described [34] using the protocol from the Puregene DNA Purification Tissue Kit (Gentra Systems, Inc., Minneapolis, MN). RNA was extracted from 17 of these micro-dissected tumor and matched normal tissue pairs using the protocol from the PureLink Micro-to-Midi Total RNA Purification System (Catalog number 12183018, Invitrogen, Carlsbad, CA). RNA quality and quantity were determined using the RNA 6000 Labchip/Agilent 2100 Bioanalyzer (Agilent Technologies, Germantown, $\mathrm{MD}$ ). The same tissue blocks were used for extraction of both DNA and RNA for each case studied.

\section{Target preparation for GeneChip Human Mapping $500 \mathrm{~K}$ array set}

The Affymetrix GeneChip Human Mapping $500 \mathrm{~K}$ array set contains $\sim 262,000$ (Nsp I array) and $\sim 238,000$ (Sty I array) SNPs (mean probe spacing $=5.8 \mathrm{~Kb}$, mean heterozygosity $=27 \%$ ). A detailed gene chip protocol can be found at http://www.affymetrix.com/support/downloads/manuals/500k_assay_manual.pdf.

Experiments were conducted according to the protocol (GeneChip Mapping Assay manual) supplied by Affymetrix, Inc. (Santa Clara, CA). Genotype calls were generated by GTYPE v 4.0 software (Affymetrix). Germline, tumor and adjacent normal DNA from each case were run together in parallel in the same experiment (ie, same batch, same day). The GEO accession numbers for these array data are GSE15526 and GSE20347.

\section{Probe preparation and hybridization for Human Genome U133A 2.0 array}

The Affymetrix Human Genome U133A 2.0 array is a single array used to interrogate expression of 14,500 well-characterized human genes. Array experiments were performed using 1-5 $\mu \mathrm{g}$ total RNA each. We followed the protocol provided by the manufacturer to carry out reverse transcription, labeling, and hybridization.

\section{GeneChip $500 \mathrm{~K}$ array data analysis}

Probe intensity data from Affymetrix $500 \mathrm{~K}$ SNP arrays were used to identify DNA alterations in the present study. To avoid gender-related issues, SNPs mapped to either the $\mathrm{X}$ or $\mathrm{Y}$ chromosome were excluded. 
Copy number $(\mathrm{CN})$ loss or gain was based on comparisons of either adjacent normal to germ-line DNA or tumor to germ-line DNA. Microarray data were first normalized using the gtype-probe set-genotype package included in Affymetrix Power Tools version 1.85. Each tumor sample was individually normalized via the BRLMM algorithm along with 99 blood samples. These blood samples were obtained from the 30 ESCC cases evaluated in the present study plus 69 healthy controls (age-, sex-, and region-matched to cases) who were all part of a larger case-control study of upper gastrointestinal cancers conducted in Shanxi Province (as noted above). Paired $\mathrm{CN}$ analysis was then performed on each sample using the Affymetrix Power Tools paired-copynumber workflow, which implements the Affymetrix Copy Number Analysis Tool (CNAT) algorithm. DNA obtained from the blood of each case served as the normal control; a sliding window of $100 \mathrm{~kb}$ was chosen to optimize the identification of extended regions of $\mathrm{CN}$ alteration (see http://www.affymetrix.com/support/technical/whitepapers/cnat_4_algorithm_whitepaper.pdf). The output of the CNAT program is $\mathrm{CN}$ state rather than an absolute $\mathrm{CN}$ prediction: normal $\mathrm{CN}$ corresponds to a state of 2 ; zero and 1 correspond to $\mathrm{CN}$ loss; and states 3 and 4 correspond to $\mathrm{CN}$ gain.

In the present study, we modified the method for identifying LOH used in our previous studies [26,27]. Here, LOH was determined using the Affymetrix Power Tools copynumber-pipeline program paired-LOH workflow. Input was *CHP files generated with the gtypeprobeset-genotype package as described above. Matched blood DNA served as the reference for $\mathrm{LOH}$ analysis for each tumor and normal adjacent sample.

\section{Combination of $\mathrm{LOH}$ and $\mathrm{CN}$ alterations}

We defined six combinations of copy number state and $\mathrm{LOH}$ status. $\mathrm{LOH}$ positive loci may have $\mathrm{CN}$ loss $(\mathrm{CN}$ $\leq 1)$, be $\mathrm{CN}$ neutral $(\mathrm{CNNLOH}, \mathrm{CN}=2)$ or show $\mathrm{CN}$ gain $(\mathrm{CN} \geq 3)$; Likewise, $\mathrm{LOH}$ negative loci may show $\mathrm{CN}$ loss, gain, or neutrality. LOH and $\mathrm{CN}$ segments for each tumor were defined independently for each sample as contiguous blocks of informative SNPs that possessed the same $\mathrm{LOH}$ and $\mathrm{CN}$ state. Endpoints of $\mathrm{LOH} / \mathrm{CN}$ segments were defined by informative SNPs. Some uninformative SNPs were located between these $\mathrm{LOH} / \mathrm{CN}$ segments; we considered these SNPs to have an undefined $\mathrm{LOH} / \mathrm{CN}$ state (see Additional file 1/Figure S1). Segment sizes were empirically observed from the data.

\section{Comparison of CN status in DNA from blood versus micro-dissected adjacent normal tissue}

DNA isolated from normal adjacent tissue is frequently used as a control in microarray experiments. In the present study we used DNA isolated from peripheral blood. We expected peripheral blood DNA to be a superior control for two reasons: first, unlike adjacent normal tissue, it is does not run the risk of being contaminated with tumor cells; second, adjacent normal tissue may actually be precancerous and contain genetic lesions. To examine whether blood DNA and adjacent normal esophageal DNA were equivalent controls, we compared copy number state calls for blood and normal adjacent from each of the 30 ESCC patients. We found that the two controls were equivalent: $99.29 \%$ to $99.99 \%$ of all copy number calls were identical. Overall, 99.96\% of SNPs in blood and $99.93 \%$ in normal adjacent tissue were $\mathrm{CN}=2$ state.

\section{Human Genome U133A 2.0 array data analysis and relation between CNNLOH and mRNA expression}

The Robust Multiarray Average (RMA) algorithm $[35,36]$ implemented in Bioconductor in R http://www. bioconductor.org was used for background correction and normalization across all samples. For each sample $\log 2$ fold changes in gene expression were calculated by subtracting the adjacent normal RMA value from the corresponding tumor RMA value.

To determine whether any gene showed a difference in the tumor versus normal gene expression fold change that was dependent on $\mathrm{LOH}$ state, we performed the following steps: (i) First, genes assayed by the U133A microarray were mapped onto each LOHCN segment of each sample. Map locations of genes were taken from the Affymetrix version na29 microarray annotation file. Note that probe sets from the same gene may have different reference sequences which differ in their chromosomal locations. Also, not every gene will map to every sample - in a particular sample, a gene may map to a gap between LOHCN regions. (ii) Next, we identified genes for which at least two of the 17 ESCC samples with expression data were $\mathrm{LOH}$ negative and at least two samples were $\mathrm{LOH}$ positive. (iii) We then performed two-sided unpaired t-tests comparing the $\log 2$ fold changes for a probe set in $\mathrm{LOH}$ positive and $\mathrm{LOH}$ negative samples. A $P$-value $<0.01$ was considered significant. (iv) Finally, SNPs on the $500 \mathrm{~K}$ microarray were mapped to the reference sequence for each expression probe set. Since probe sets from the same gene may have different reference sequences, they may differ in the number of SNPs assigned to them (Additional file 2/Figure S2).

\section{Results}

In the present study we determined copy number and loss of heterozygosity $(\mathrm{LOH})$ status in DNA isolated from germ-line and micro-dissected tumor and matched adjacent normal samples from 30 ESCC patients using 
the Affymetrix $500 \mathrm{~K} \mathrm{SNP}$ array. The average genotype call rate was $96 \%$ (89-99\%): the $250 \mathrm{~K}$ Nsp I array was 96\% (90-98\%) and $250 \mathrm{~K}$ Sty I array was 95\% (89-99\%). Genotype call rates were similar for all three tissue types examined. We first analyzed whether copy numbers were similar between DNAs from the two normal tissues: germ-line (blood) and micro-dissected adjacent normal samples. Our analysis indicated that DNA CN values were similar between the two normal tissues (Additional file 3 - Table S1), as expected. Our results indicate that germ-line DNA can be used as a normal control in studies of $\mathrm{CN}$ alteration; it is more readily available than matched adjacent normal tissue.

\section{Complex DNA alterations in ESCC}

The distribution of DNA alterations in each of the 30 ESCC cases is summarized in Table 1 (with $\mathrm{LOH}$ ) and in Additional file 4/Table S2 (without $\mathrm{LOH}$ ). We divided genomic regions into three groups based on $\mathrm{CN}$ states: $\mathrm{CN}$ loss, neutral, and gain. We found that $50 \%, 90 \%$, and 93\% of cases showed $\mathrm{LOH}$ in the $\mathrm{CN}$ loss, neutral, and gain groups, respectively (Table 1 ). For each chromosome, we also calculated the percentage of SNPs involved in LOH for each group. They ranged between $20-57 \%$, 7 $100 \%$, and $2-100 \%$ for the CN loss, neutral, and gain groups, respectively (Table 1 ). Our results suggest that $\mathrm{LOH}$ with $\mathrm{CN}$ neutral or gain are common phenomena

Table 1 LOH by copy number in ESCC cases by individual case ( $\mathbf{N}=\mathbf{3 0}$ )

\begin{tabular}{|c|c|c|c|c|}
\hline Case ID & $\begin{array}{r}\text { Total no. informative } \\
\text { SNPs with LOH }\end{array}$ & $\begin{array}{r}\text { No. informative SNPs } \\
\text { with LOH and CN = } 1 \text { (fraction) }\end{array}$ & $\begin{array}{l}\text { No. informative SNPs with } \\
\text { LOH and CN }=2 \text { (fraction) }\end{array}$ & $\begin{array}{r}\text { No. informative SNPs with } \\
\mathrm{LOH} \text { and } \mathrm{CN}=3 \text { or } 4 \text { (fraction) }\end{array}$ \\
\hline$* 1$ & 31,808 & $2,260(0.07)$ & $26,801(0.84)$ & $2,747(0.09)$ \\
\hline 2 & 368 & $0(0)$ & $205(0.56)$ & $163(0.44)$ \\
\hline 3 & 377 & $0(0)$ & $139(0.37)$ & $238(0.63)$ \\
\hline$* 4$ & 36,175 & 2,093 (0.06) & $27,655(0.76)$ & $6,427(0.18)$ \\
\hline$*_{5}$ & 24 & $0(0)$ & $0(0)$ & $24(1.00)$ \\
\hline$* 6$ & 14,751 & $210(0.01)$ & $12,661(0.86)$ & $1,880(0.13)$ \\
\hline$* 7$ & 3,559 & $0(0)$ & $2,905(0.82)$ & $654(0.18)$ \\
\hline${ }^{*} 8$ & 2,408 & $4(0)$ & $2,217(0.92)$ & $187(0.08)$ \\
\hline${ }^{*} 9$ & 593 & $1(0)$ & $266(0.45)$ & $326(0.55)$ \\
\hline 10 & 17,546 & $1,075(0.06)$ & $12,087(0.69)$ & $4,384(0.25)$ \\
\hline 11 & 78,159 & $3,678(0.05)$ & $67,726(0.87)$ & $6,755(0.09)$ \\
\hline 12 & 41 & $0(0)$ & $0(0)$ & $41(1.00)$ \\
\hline${ }^{*} 13$ & 6,113 & $209(0.03)$ & $5,154(0.84)$ & $750(0.12)$ \\
\hline 14 & 13,498 & $4,084(0.30)$ & $9,190(0.68)$ & $224(0.02)$ \\
\hline 15 & 3 & $0(0)$ & $3(1.00)$ & $0(0)$ \\
\hline 16 & 1431 & $0(0)$ & $54(0.04)$ & $1,377(0.96)$ \\
\hline 17 & 16,934 & $5,732(0.34)$ & $10,842(0.64)$ & $360(0.02)$ \\
\hline 18 & 2,107 & $0(0)$ & $1,553(0.74)$ & $554(0.26)$ \\
\hline${ }^{*} 19$ & 0 & $0(0)$ & $0(0)$ & $0(0)$ \\
\hline${ }^{*} 20$ & 527 & $0(0)$ & $137(0.26)$ & $390(0.74)$ \\
\hline$* 21$ & 19,954 & $62(0)$ & $16,332(0.82)$ & $3,560(0.18)$ \\
\hline$* 22$ & 11,180 & $4,410(0.39)$ & $6,357(0.57)$ & $413(0.04)$ \\
\hline$* 23$ & 14,523 & $5,939(0.41)$ & $5,953(0.41)$ & $2,631(0.18)$ \\
\hline 24 & 15,773 & $5,672(0.36)$ & $9,354(0.59)$ & $747(0.05)$ \\
\hline${ }^{*} 25$ & 23,005 & $20(0)$ & $17,114(0.74)$ & $5,871(0.26)$ \\
\hline$* 26$ & 37,691 & $21,495(0.57)$ & $15,229(0.40)$ & $967(0.03)$ \\
\hline 27 & 12,120 & $4,500(0.37)$ & $6,299(0.52)$ & $1,321(0.11)$ \\
\hline 28 & 494 & $0(0)$ & $37(0.07)$ & $457(0.93)$ \\
\hline$* 29$ & 18,222 & $444(0.02)$ & $15,400(0.85)$ & $2,378(0.13)$ \\
\hline$* 30$ & 42,499 & $16,432(0.39)$ & $25,394(0.60)$ & $673(0.02)$ \\
\hline \multicolumn{2}{|c|}{ No. cases with SNP LOH fraction $=0$} & 15 & 3 & 2 \\
\hline \multicolumn{2}{|c|}{ Range (fraction SNPs with $\mathrm{LOH}$ ) } & $0-0.57$ & $0-1.00$ & $0-1.00$ \\
\hline \multicolumn{2}{|c|}{ Median (fraction SNPs with LOH) } & 0.00 & 0.60 & 0.13 \\
\hline \multicolumn{2}{|c|}{ Global average (fraction SNPs with LOH) } & 0.19 & 0.70 & 0.11 \\
\hline
\end{tabular}

${ }^{*}$ Case also examined with Affymetrix U133A 2.0 chip. 
in ESCC. For SNPs without LOH, we also calculated the percent of SNPs in each CN state; averages were $5 \%$, $84 \%$, and $11 \%$ for $\mathrm{CN}$ loss, neutral, and gain, respectively.

The distribution of the six types of DNA alterations for all 30 cases by chromosome arm is shown in Table 2 (with $\mathrm{LOH}$ ) and Additional file 5/Table S3 (without $\mathrm{LOH}$ ). CNNLOH was observed on all chromosome arms, but most frequently on $19 \mathrm{p}$ (100\%), 5p (96\%), 2p (95\%), and $20 \mathrm{q}(95 \%)$. The highest frequencies of $\mathrm{LOH}$ with $\mathrm{CN}$ loss $(\mathrm{CN}=1)$ were found on $3 \mathrm{p}(56 \%), 5 \mathrm{q}(47 \%)$, and $21 \mathrm{q}$ (41\%); relatively high frequencies were also seen on $18 \mathrm{q}$ (31\%), 11q (29\%), 1p (28\%), 19q (27\%), and 11p (25\%). $\mathrm{LOH}$ with $\mathrm{CN}$ gain was most common on 20p (82\%), 8q $(74 \%)$ and $3 q(42 \%)$ (Table 2 and Figure 1). Taken

Table 2 LOH by copy number in ESCC cases by chromosomal arm ( $\mathrm{N}=30$ cases)

\begin{tabular}{|c|c|c|c|c|}
\hline $\begin{array}{r}\text { Chromosomal } \\
\text { arm }\end{array}$ & $\begin{array}{r}\text { Total no. informative } \\
\text { SNPs with LOH }\end{array}$ & $\begin{array}{l}\text { No. informative SNPs with } \\
\text { LOH and CN = } 1 \text { (fraction) }\end{array}$ & $\begin{array}{l}\text { No. informative SNPs with } \\
\text { LOH and CN }=2 \text { (fraction) }\end{array}$ & $\begin{array}{r}\text { No. informative SNPs with LOH } \\
\text { and } \mathrm{CN}=3 \text { or } 4 \text { (fraction) }\end{array}$ \\
\hline $1 p$ & 10,576 & $2,930(0.28)$ & $6,522(0.62)$ & $1,124(0.11)$ \\
\hline $1 q$ & 8,366 & $459(0.05)$ & $7,674(0.92)$ & $233(0.03)$ \\
\hline $2 p$ & 11,321 & $371(0.03)$ & $10,770(0.95)$ & $180(0.02)$ \\
\hline $2 q$ & 23,115 & $3,015(0.13)$ & $18,939(0.82)$ & $1,161(0.05)$ \\
\hline $3 p$ & 27,593 & $15,335(0.56)$ & $12,064(0.44)$ & $194(0.01)$ \\
\hline $3 q$ & 7,256 & $41(0.01)$ & $4,168(0.57)$ & $3,047(0.42)$ \\
\hline $4 p$ & 12,223 & 4,771 (0.39) & $7,452(0.61)$ & $0(0)$ \\
\hline $4 q$ & 28,105 & $9,142(0.33)$ & $17,905(0.64)$ & $1,958(0.04)$ \\
\hline $5 p$ & 1,315 & $0(0)$ & $1,259(0.96)$ & $56(0.04)$ \\
\hline $5 q$ & 16,537 & $7,744(0.47)$ & $8,777(0.53)$ & $16(0)$ \\
\hline $6 p$ & 5,622 & $342(0.06)$ & $4,870(0.87)$ & $410(0.07)$ \\
\hline $6 q$ & 3,365 & $165(0.05)$ & $2,773(0.82)$ & $427(0.13)$ \\
\hline $7 p$ & 7,389 & $9(0)$ & $5,491(0.74)$ & $1,889(0.26)$ \\
\hline $7 q$ & 8,001 & $151(0.02)$ & $6,452(0.74)$ & $1,398(0.17)$ \\
\hline $8 p$ & 8,803 & $1,580(0.18)$ & $6,234(0.71)$ & $989(0.11)$ \\
\hline $8 q$ & 17,321 & $47(0)$ & 4,633 (0.27) & $12,641(0.74)$ \\
\hline $9 p$ & 18,292 & $2,772(0.15)$ & $13,333(0.73)$ & $2,187(0.12)$ \\
\hline $9 q$ & 31,400 & $1,965(0.06)$ & $27,212(0.87)$ & $2,223(0.07)$ \\
\hline $10 p$ & 2,739 & $472(0.17)$ & $2,063(0.75)$ & $204(0.07)$ \\
\hline $10 q$ & 14,651 & $1,728(0.12)$ & $12,075(0.82)$ & $848(0.06)$ \\
\hline $11 p$ & 9,391 & $2,367(0.25)$ & $6,902(0.73)$ & $122(0.01)$ \\
\hline $11 q$ & 17,377 & $5,116(0.29)$ & $10,422(0.60)$ & $1,839(0.11)$ \\
\hline $12 p$ & 5,229 & $156(0.03)$ & $3,251(0.62)$ & $1,822(0.34)$ \\
\hline $12 q$ & 6,794 & $67(0.01)$ & $5,980(0.88)$ & $747(0.11)$ \\
\hline $13 q$ & 35,648 & $5,897(0.17)$ & $25,964(0.73)$ & $3,787(0.11)$ \\
\hline $14 q$ & 10,931 & $1,484(0.14)$ & $7,446(0.68)$ & $2,001(0.18)$ \\
\hline $15 q$ & 10,194 & $685(0.07)$ & $8,313(0.82)$ & $1,196(0.12)$ \\
\hline $16 p$ & 1,395 & $111(0.08)$ & $1,284(0.92)$ & $0(0)$ \\
\hline $16 q$ & 3,127 & $1(0)$ & 2,832 (0.91) & $294(0.09)$ \\
\hline $17 p$ & 7,719 & $324(0.04)$ & $6,939(0.90)$ & $456(0.06)$ \\
\hline $17 q$ & 16,328 & $38(0)$ & $14,754(0.90)$ & $1,536(0.09)$ \\
\hline $18 p$ & 2,596 & $488(0.19)$ & $1,147(0.44)$ & $961(0.37)$ \\
\hline $18 q$ & 9,978 & $3,108(0.31)$ & $6,838(0.69)$ & $32(0)$ \\
\hline $19 p$ & 1,069 & $1(0)$ & $1,065(1.00)$ & $3(0)$ \\
\hline $19 q$ & 3,505 & $932(0.27)$ & $2,482(0.71)$ & $91(0.03)$ \\
\hline $20 p$ & 1,278 & $0(0)$ & $229(0.18)$ & $1,049(0.82)$ \\
\hline $20 q$ & 1,773 & $60(0.03)$ & $1,677(0.95)$ & $36(0.02)$ \\
\hline $21 q$ & 9,444 & $3,825(0.41)$ & $5,599(0.59)$ & $20(0)$ \\
\hline $22 q$ & 4,177 & $621(0.15)$ & $3,274(0.80)$ & $222(0.05)$ \\
\hline \multicolumn{2}{|c|}{ Range (fraction SNPs with LOH) } & $0-0.56$ & $0.18-1.00$ & $0-0.82$ \\
\hline \multicolumn{2}{|c|}{ Median (fraction SNPs with LOH) } & 0.05 & 0.71 & 0.05 \\
\hline \multicolumn{2}{|c|}{ Global average (fraction SNPs with LOH) } & 0.19 & 0.70 & 0.11 \\
\hline
\end{tabular}




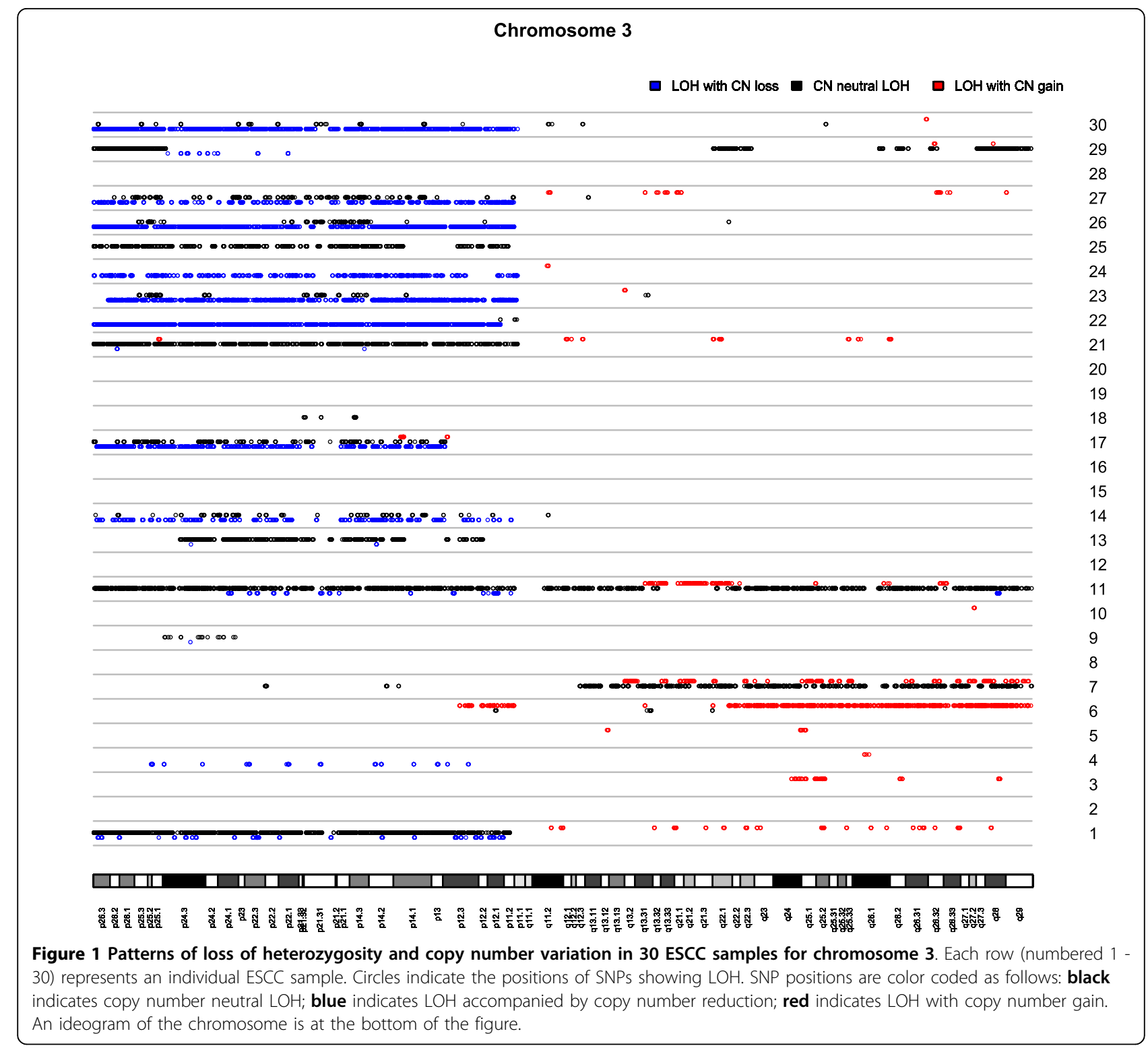

together, our results show that $\mathrm{LOH}$ with $\mathrm{CNN}$ or $\mathrm{CN}$ gain were much more frequent than $\mathrm{LOH}$ with $\mathrm{CN}$ loss on every chromosome arm but one (ie, 3p).

Results of $\mathrm{CN}$ alterations in non-LOH group by chromosome arms are summarized in Additional file 5/ Table S3. Briefly, a frequency of CN loss $\geq 10 \%$ was observed on eight chromosome arms (3p, 4p, 4q, 5q, 8p, $9 \mathrm{p}, 11 \mathrm{q}$, and 13q). A frequency of $\mathrm{CN}$ gain $\geq 10 \%$ was observed on 13 chromosome arms (1q, 2p, 2q 3q, 5p, $7 \mathrm{p}, 7 \mathrm{q}, 8 \mathrm{q}, 12 \mathrm{p}, 14 \mathrm{q}, 18 \mathrm{p}, 20 \mathrm{p}$, and 20q).

\section{Relation between genomic alterations and gene expression}

The average present call rate on the Human Genome $\mathrm{U} 133 \mathrm{~A}$ array was $53 \%$ (range $51-61 \%$ ) for the 34 chips from the 17 sample pairs with sufficient tissue for RNA isolation and testing. To investigate the relation between $\mathrm{LOH} / \mathrm{CNV}$ and gene expression levels, we intersected genes on the Affymetrix U133A chip with SNPs on the $500 \mathrm{~K} \mathrm{SNP}$ array. SNPs that mapped within genes are summarized in Additional file 6/Table S4 and include 169,687 SNPs within 12,225 genes.

We were interested in identifying differentiallyexpressed genes between $\mathrm{LOH}$ and non-LOH groups in genes that were $\mathrm{CN}$ neutral. A total of 4,572 genes qualified for this analysis (see Methods). Among these genes, 168 genes showed significant differences in expression between tumors with and without LOH $(P<0.01)$ (Additional file 7/Table S5). Based on chance alone (at the $P<$ 0.01 level), differences in only 45 genes would be 
Table 3 Comparison of gene expression in copy number neutral (CNN) genes with LOH and without LOH (normal) ( $\mathrm{N}=$ 46 genes significantly differentially-expressed 2 -fold or greater)*

\begin{tabular}{|c|c|c|c|c|c|c|c|c|c|}
\hline $\begin{array}{l}\text { Gene } \\
\text { name }\end{array}$ & Cytoband & $\begin{array}{l}\text { Probeset on } \\
\text { U133A array }\end{array}$ & $\begin{array}{l}\text { No. cases } \\
\text { with LOH }\end{array}$ & $\begin{array}{c}\text { No. cases } \\
\text { without } \mathrm{LOH}\end{array}$ & $\begin{array}{l}\text { No. } \\
\text { SNPs }\end{array}$ & $\begin{array}{l}\text { Fold change } \\
\text { CNN with LOH }\end{array}$ & $\begin{array}{c}\text { Fold change CNN } \\
\text { without } \mathrm{LOH}\end{array}$ & T-test & P-value \\
\hline
\end{tabular}

Genes where expression level of CNN with $L O H$ is less than $C N N$ without $L O H$ ( $N=28$ genes)

\begin{tabular}{|c|c|c|c|c|c|c|c|c|c|}
\hline ADK & $\begin{array}{l}\text { chr10q22 } \\
10 q 11-q 24\end{array}$ & 204120_s_at & 2 & 13 & 46 & 0.404 & 0.606 & 3.138 & 8.559E-03 \\
\hline ADK & $\begin{array}{l}\text { chr10q22 } \\
10 q 11-q 24\end{array}$ & 204119_s_at & 2 & 13 & 46 & 0.411 & 0.619 & 3.195 & 7.278E-03 \\
\hline AIM1L & chr1p36.11 & 220289_s_at & 2 & 15 & 6 & 0.093 & 0.201 & 3.548 & $4.812 \mathrm{E}-03$ \\
\hline APOL6 & chr22q12.3 & 219716_at & 2 & 5 & 4 & 0.467 & 0.894 & 4.659 & 9.543E-03 \\
\hline ATP6VOE1 & chr5q35.2 & 201171_at & 2 & 12 & 5 & 0.375 & 0.669 & 3.234 & 9.843E-03 \\
\hline ATP6VOE1 & chr5q35.2 & 200096_s_at & 2 & 12 & 5 & 0.480 & 0.872 & 4.445 & 5.699E-03 \\
\hline BACH1 & chr21q22.11 & 204194_at & 2 & 12 & 9 & 0.498 & 0.738 & 3.970 & 2.098E-03 \\
\hline BTG2 & chr1q32 & 201236_s_at & 2 & 14 & 1 & 0.460 & 0.986 & 4.095 & 1.183E-03 \\
\hline $\mathrm{CD} 2$ & chr1p13.1 & 205831_at & 2 & 14 & 1 & 0.324 & 0.628 & 3.462 & $9.221 \mathrm{E}-03$ \\
\hline CEACAM6 & chr19q13.2 & 211657_at & 2 & 13 & 3 & 0.007 & 0.100 & 4.779 & 7.023E-03 \\
\hline CRAT & chr9q34.1 & 209522_s_at & 7 & 8 & 1 & 0.293 & 0.567 & 3.169 & 7.495E-03 \\
\hline CSDE1 & chr1p22 & 202646_s_at & 2 & 14 & 3 & 0.343 & 0.895 & 7.835 & 7.590E-05 \\
\hline CYP4F3 & chr19p13.2 & 206515_at & 2 & 13 & 136 & 0.065 & 0.307 & 3.329 & $5.631 \mathrm{E}-03$ \\
\hline $\mathrm{D} / \mathrm{O} 2$ & $\begin{array}{c}\text { chr14q24.2- } \\
\text { q24.3 }\end{array}$ & 203699_s_at & 2 & 11 & 4 & 0.072 & 0.127 & 3.565 & $6.175 \mathrm{E}-03$ \\
\hline $\mathrm{D} / \mathrm{O} 2$ & $\begin{array}{c}\text { chr14q24.2- } \\
\text { q24.3 }\end{array}$ & 203700_s_at & 2 & 11 & 4 & 0.168 & 0.280 & 3.697 & $3.752 \mathrm{E}-03$ \\
\hline EIF4EBP2 & $\begin{array}{c}\text { chr10q21- } \\
\text { q22 }\end{array}$ & 208770_s_at & 2 & 14 & 1 & 0.452 & 0.657 & 6.300 & 2.692E-05 \\
\hline FAS & chr10q24.1 & 216252_x_at & 2 & 13 & 8 & 0.412 & 0.648 & 3.058 & 9.883E-03 \\
\hline HERC6 & chr4q22.1 & 219352_at & 3 & 9 & 1 & 0.105 & 0.538 & 5.769 & 2.901E-04 \\
\hline ICAM3 & $\begin{array}{c}\text { chr19p13.3- } \\
\text { p13.2 }\end{array}$ & 204949_at & 2 & 13 & 2 & 0.356 & 0.602 & 3.478 & 4.342E-03 \\
\hline ILIRN & chr2q14.2 & 212659_s_at & 2 & 12 & 13 & 0.073 & 0.142 & 3.256 & 7.793E-03 \\
\hline NADSYN1 & chr11q13.4 & 218840_s_at & 2 & 8 & 10 & 0.448 & 0.649 & 3.399 & 9.384E-03 \\
\hline NHP2 & chr5q35.3 & 216583_x_at & 2 & 10 & 1 & 0.497 & 0.774 & 4.091 & $2.584 \mathrm{E}-03$ \\
\hline PDCD4 & chr10q24 & 202730_s_at & 2 & 12 & 4 & 0.212 & 0.363 & 4.483 & $9.256 \mathrm{E}-04$ \\
\hline PPP1R13L & chr19q13.32 & 218849_s_at & 3 & 13 & 1 & 0.190 & 0.487 & 5.016 & $5.018 \mathrm{E}-04$ \\
\hline RIPK4 & chr21q22.3 & 221215_s_at & 5 & 10 & 7 & 0.217 & 0.454 & 3.614 & $3.658 \mathrm{E}-03$ \\
\hline SKAP2 & chr7p21-p15 & 216899_s_at & 2 & 10 & 12 & 0.293 & 0.615 & 4.165 & 1.973E-03 \\
\hline SKAP2 & chr7p21-p15 & 204361_s_at & 2 & 10 & 43 & 0.359 & 0.665 & 4.384 & $1.591 \mathrm{E}-03$ \\
\hline SLC6A1 & chr3p25-p24 & 205152_at & 2 & 8 & 6 & 0.158 & 0.438 & 5.044 & $2.481 \mathrm{E}-03$ \\
\hline STK39 & chr2q24.3 & 202786_at & 2 & 12 & 86 & 0.151 & 0.290 & 3.363 & $6.327 \mathrm{E}-03$ \\
\hline SYNPOZL & chr10q22.2 & 219804_at & 3 & 13 & 1 & 0.063 & 0.345 & 6.925 & 8.703E-06 \\
\hline TGM5 & chr15q15.2 & 207911_s_at & 2 & 13 & 8 & 0.382 & 0.517 & 3.496 & 4.389E-03 \\
\hline ZNF91 & $\begin{array}{c}\text { chr19p13.1- } \\
\text { p12 }\end{array}$ & 206059_at & 2 & 10 & 5 & 0.026 & 0.551 & 9.054 & $6.430 \mathrm{E}-04$ \\
\hline
\end{tabular}

Genes where expression level of CNN with $L O H$ is greater than $C N N$ without $L O H$ ( $N=18$ genes)

\begin{tabular}{|c|c|c|c|c|c|c|c|c|c|}
\hline ACVR1 & chr2q23-q24 & 203935_at & 2 & 12 & 13 & 3.480 & 1.751 & -4.533 & 7.869E-04 \\
\hline ASPN & chr9q22 & 219087_at & 5 & 10 & 2 & 10.594 & 2.679 & -3.792 & $2.667 \mathrm{E}-03$ \\
\hline$B \cup B 1$ & chr2q14 & 209642_at & 2 & 12 & 2 & 8.571 & 4.010 & -3.922 & $2.170 \mathrm{E}-03$ \\
\hline CALU & chr7q32.1 & 200757_s_at & 2 & 12 & 7 & 3.467 & 1.830 & -4.612 & 7.505E-04 \\
\hline CALU & chr7q32.1 & 200755_s_at & 2 & 12 & 7 & 3.899 & 1.981 & -3.903 & 2.269E-03 \\
\hline CAMSAP1L1 & chr1q32.1 & 212765_at & 2 & 13 & 3 & 3.000 & 1.357 & -6.288 & $1.629 \mathrm{E}-03$ \\
\hline CENPF & chr1q32-q41 & 209172_s_at & 2 & 14 & 10 & 5.481 & 2.643 & -5.909 & $3.926 \mathrm{E}-05$ \\
\hline CSNKIE & chr22q13.1 & 202332_at & 2 & 3 & 4 & 2.933 & 1.157 & -7.434 & $9.278 \mathrm{E}-03$ \\
\hline
\end{tabular}


Table 3: Comparison of gene expression in copy number neutral (CNN) genes with LOH and without LOH (normal) (N = 46 genes significantly differentially-expressed 2 -fold or greater)* (Continued)

\begin{tabular}{|c|c|c|c|c|c|c|c|c|c|}
\hline FAM13A & chr4q22.1 & 202973_x_at & 3 & 10 & 14 & 2.756 & 1.149 & -3.508 & $6.115 \mathrm{E}-03$ \\
\hline FHL2 & chr2q12-q14 & 202949_s_at & 2 & 14 & 15 & 3.159 & 1.244 & -4.697 & 4.097E-04 \\
\hline GSR & chr8p21.1 & 205770_at & 3 & 10 & 8 & 2.505 & 1.222 & -3.701 & 4.824E-03 \\
\hline ITGA6 & chr2q31.1 & 201656_at & 2 & 11 & 21 & 4.038 & 2.400 & -3.519 & 4.820E-03 \\
\hline KIF14 & chr1q32.1 & 206364_at & 2 & 13 & 6 & 11.027 & 3.578 & -8.257 & $2.071 \mathrm{E}-06$ \\
\hline NCAPH & chr2q11.2 & 212949_at & 2 & 11 & 2 & 3.643 & 1.943 & -5.087 & 4.450E-04 \\
\hline NRP2 & chr2q33.3 & 214632_at & 3 & 11 & 29 & 2.161 & 1.257 & -3.500 & $9.276 \mathrm{E}-03$ \\
\hline RBM28 & chr7q32.1 & 218593_at & 2 & 11 & 9 & 2.210 & 1.555 & -3.976 & $2.370 \mathrm{E}-03$ \\
\hline SMAD5 & chr5q31 & 205187_at & 2 & 12 & 3 & 2.208 & 0.829 & -10.692 & $3.171 \mathrm{E}-07$ \\
\hline STEAP3 & chr2q14.2 & 218424_s_at & 2 & 14 & 12 & 2.707 & 1.207 & -6.922 & $9.985 \mathrm{E}-06$ \\
\hline TGS1 & chr8q11 & 219231_at & 2 & 8 & 1 & 2.533 & 1.656 & -3.495 & $8.601 \mathrm{E}-03$ \\
\hline
\end{tabular}

*Sorted alphabetically by gene name.

expected, therefore, expression differences were observed in over three times as many genes as expected. One hundred and one (60\%) of the 168 genes showed lower expression levels in $\mathrm{CNNLOH}$ than in the normal group (ie, CNN, no LOH), whereas 67 genes (40\%) showed higher expression levels in CNNLOH (Additional file 7/ Table S5). Twenty-eight of the 101 down-regulated genes (32 probes) and 18 of the 67 up-regulated genes (19 probes) showed expression differences $\geq 2$-fold (Table 3 ). These findings suggest that in the $\mathrm{CN}$ neutral state, $\mathrm{LOH}$ can affect gene expression.

We also compared expression of genes with $\mathrm{LOH}$ versus no $\mathrm{LOH}$ in $\mathrm{CN}$ loss genes. We identified six of 600 genes which showed significantly different expression between the LOH groups. All six genes showed increased expression in tumors with LOH (Table 4a).
Finally, we compared gene expression in the $\mathrm{CN}$ gain state between tumors with and without LOH. We found that six of 354 genes showed significant differences in expression between the two groups, including two down-regulated and four up-regulated genes (Table 4b).

\section{Discussion}

We characterized ESCC tumors for complex DNA alterations - LOH and CNV - and related these genomic alterations to gene expression. To our knowledge, this is the first report to comprehensively address the distribution of complex DNA alterations in ESCC and its relation to gene expression on a genome-wide scale.

Ninety percent of cases showed $\mathrm{CNNLOH}$ in their tumors and, over all cases, CNNLOH was found on

Table 4 Comparison of gene expression in copy number loss/gain genes with LOH and without LOH*

\begin{tabular}{|c|c|c|c|c|c|c|c|c|c|}
\hline $\begin{array}{l}\text { Gene } \\
\text { name }\end{array}$ & Cytoband & $\begin{array}{l}\text { Probeset on } \\
\text { U133A array }\end{array}$ & $\begin{array}{l}\text { No. cases } \\
\text { with LOH }\end{array}$ & $\begin{array}{c}\text { No. cases } \\
\text { without LOH }\end{array}$ & $\begin{array}{l}\text { No. } \\
\text { SNPs }\end{array}$ & $\begin{array}{l}\text { Fold change } \\
\text { with } \mathrm{LOH}\end{array}$ & $\begin{array}{l}\text { Fold change } \\
\text { without LOH }\end{array}$ & T-test & P-value \\
\hline
\end{tabular}

Table 4.a.: Genes/probes with $C N$ loss $(C N=1)$

\begin{tabular}{cccccccccc}
\hline ATXN7 & chr3p21.1-p12 & 204516_at & 4 & 2 & 10 & 0.574 & 0.258 & -8.233 & $1.333 \mathrm{E}-03$ \\
CTDSPL & chr3p21.3 & 213597_s_at & 3 & 3 & 13 & 0.997 & 0.890 & -5.414 & $8.188 \mathrm{E}-03$ \\
F2R & chr5q13 & 203989_x_at & 3 & 2 & 4 & 6.083 & 1.217 & -6.771 & $7.275 \mathrm{E}-03$ \\
RAB5A & chr3p24-p22 & 209089_at & 5 & 2 & 6 & 0.419 & 0.240 & -9.065 & $5.698 \mathrm{E}-04$ \\
RAF1 & chr3p25 & 201244_s_at & 3 & 2 & 9 & 0.555 & 0.389 & -24.008 & $5.074 \mathrm{E}-04$ \\
SCN10A & chr3p22-p21 & 208578_at & 3 & 4 & 23 & 1.806 & 0.884 & -4.253 & $8.107 \mathrm{E}-03$ \\
\hline
\end{tabular}

Table 4.b.: Genes/probes with $C N$ gain (CN $=3$ or 4$)$

\begin{tabular}{cccccccccc}
\hline ARHGAP5 & chr14q12 & 217936_at & 2 & 5 & 1 & 1.731 & 0.769 & -4.809 & $8.159 \mathrm{E}-03$ \\
CYP11B1 & chr8q21 & 214610_at & 2 & 6 & 3 & 0.915 & 1.114 & 4.728 & $3.469 \mathrm{E}-03$ \\
NFATC4 & chr14q11.2 & 205897_at & 2 & 3 & 1 & 1.363 & 0.903 & -11.692 & $2.823 \mathrm{E}-03$ \\
PAX9 & chr14q12-q13 & 207059_at & 2 & 5 & 1 & 0.898 & 0.319 & -5.395 & $5.706 \mathrm{E}-03$ \\
PPFIA1 & chr11q13.3 & 202066_at & 7 & 2 & 12 & 7.524 & 2.448 & -3.918 & $6.425 \mathrm{E}-03$ \\
TP63 & chr3q28 & 211834_s_at & 2 & 13 & 17 & 0.901 & 1.197 & 3.438 & $9.809 \mathrm{E}-03$ \\
TP63 & chr3q28 & 211194_s_at & 2 & 13 & 18 & 0.987 & 2.649 & 4.008 & $3.640 \mathrm{E}-03$ \\
\hline
\end{tabular}


every chromosome arm, indicating that it is a common phenomenon.

The frequency of CNNLOH observed here in ESCC was much less than has been reported in other cancers [3-19]. For example, in colon cancer and basal cell carcinoma nearly all $\mathrm{LOH}$ was associated with copy number neutral regions $[3,10]$. In general, CNNLOH occurs with variable frequency in different genomic regions in tumors of different origin. There are several differences between the study reported here and previous studies which likely influenced the results. First, DNA from micro-dissected tumor and adjacent normal was used in the present study, while either cancer DNA without matched controls or cancer cell lines were used in most other reported studies. Second, we examined $\mathrm{LOH}$ and $\mathrm{CN}$ alterations using the same SNP array platform, while other studies used SNPs for LOH and CGH arrays for $\mathrm{CN}$ analyses. Third, the criteria for identifying $\mathrm{LOH}$ differed among the studies reported. Finally, the types of cancers studied previously differ from the present study which is the first report of CNNLOH in ESCC.

In previous $\mathrm{LOH}$ studies, we reported high-frequency LOH on several chromosome arms, including $3 \mathrm{p}, 4 \mathrm{p}$, 4q, 9p, 9q, 13q, 17p, and 17q [23,26,27]. By integrating $\mathrm{LOH}$ and $\mathrm{CN}$ alteration data in the present study, we can now say that the $\mathrm{LOH}$ on $3 p$ is primarily due to $\mathrm{CN}$ loss $\mathrm{LOH}$, while the $\mathrm{LOH}$ on the other seven chromosome arms is predominantly due to CNNLOH.

Our results showed that $\mathrm{CNNLOH}$ can change expression levels of genes in ESCC, either increasing or decreasing them. We do not know why CNNLOH changes gene expression, but one possibility is that the two alleles may have different gene expression levels. For example, if allele $\mathrm{A}$ expression is greater than allele $\mathrm{B}$, the expression level for the 3 genotypes would be ordered as $A A>A B>$ BB. CNNLOH with retention of two B alleles (genotype $\mathrm{BB}$ ) would then show lower expression than genotype $\mathrm{AB}$. Conversely, CNNLOH with loss of the allele B would result in two copies of allele $A$ and a higher level of expression than that of $\mathrm{AB}$ cells. Another possibility is that the two alleles have different expression due to different epigenetic states, with $\mathrm{LOH}$ resulting in copies with two extreme epigenetic states. A third possibility is that one allele harbors a mutation and subsequent $\mathrm{LOH}$ leads to a homozygous mutant. Several studies have shown that $\mathrm{CNNLOH}$ regions can harbor mutated genes. For example, JAK2 V617F, FLT3-ITD, AML1/RUNX1, WT1, and NPM1 mutations were all found in CNNLOH regions in AML [15]. These various hypotheses merit testing in the future.

The study design in the present study has several important features: (i) we compared $\mathrm{CN}$ status between DNA from germ-line and micro-dissected adjacent normal tissue; (ii) we used micro-dissected DNA from tumor tissue; (iii) we assessed both $\mathrm{LOH}$ and $\mathrm{CN}$ alterations simultaneously using the same array platform; and (iv) we integrated complex DNA alterations and gene expression data on a genome-wide level using both high density SNP and expression arrays in the same cases. A noteworthy weakness of our study is the relatively small number of cases evaluated (including a particularly small number of cases with both $\mathrm{LOH}$ and RNA expression data to evaluate, due in part to the $500 \mathrm{~K}$ chip mean heterozygosity of $27 \%$ ), which limited our power to detect significant differences in loci between $\mathrm{LOH}$ and non-LOH groups. In addition, findings for ESCC from this high-risk region may not be generalizable to populations elsewhere in the world.

In summary, we investigated the distribution of complex DNA alterations in ESCCs at the genome-wide level and determined that $\mathrm{CN}$ neutral is the most common $\mathrm{CN}$ state in $\mathrm{LOH}$, and that $\mathrm{CNNLOH}$ is a very common phenomenon overall. Importantly, we also showed that CNNLOH could alter the expression level of genes affected in ESCC.

\section{Conclusion}

$\mathrm{CNNLOH}$ is a common phenomenon in many cancers, including ESCC, and non-disjunction and/or somatic recombination are the most likely mechanisms for its occurrence. $\mathrm{CNNLOH}$ can result in changes in gene expression which are functionally significant. Expression differences in $\mathrm{CNNLOH}$ suggest that alleles are different in terms of their gene expression potential, and that these differences may result from differences in genotype and/or epigenetics.

\section{Additional material}

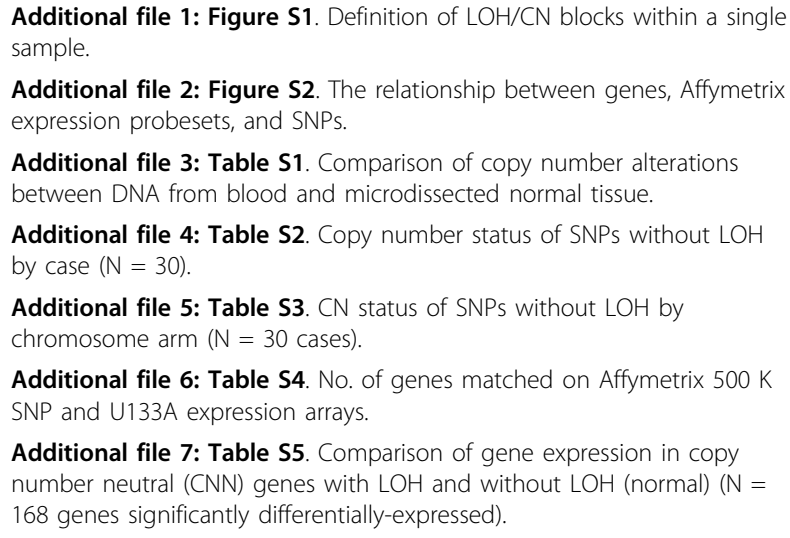

Additional file 2: Figure S2. The relationship between genes, Affymetrix expression probesets, and SNPS.

Additional file 3: Table S1. Comparison of copy number alterations between DNA from blood and microdissected normal tissue.

Additional file 4: Table S2. Copy number status of SNPs without $\mathrm{LOH}$ by case $(\mathrm{N}=30)$.

Additional file 5: Table S3. CN status of SNPS without LOH by chromosome $\operatorname{arm}(\mathrm{N}=30$ cases)

Additional file 6: Table S4. No. of genes matched on Affymetrix $500 \mathrm{~K}$ SNP and U133A expression arrays.

Additional file 7: Table S5. Comparison of gene expression in copy number neutral (CNN) genes with $\mathrm{LOH}$ and without $\mathrm{LOH}$ (normal) $(\mathrm{N}=$ 168 genes significantly differentially-expressed).

\section{Abbreviations}

SNP: single nucleotide polymorphism; ESCC: esophageal squamous cell carcinoma; (CNNLOH): copy number neutral loss of heterozygosity; CN: copy number. 


\section{Acknowledgements}

This research was supported by the Intramural Research Program of the $\mathrm{NIH}$ the National Cancer Institute, the Division of Cancer Epidemiology and Genetics, and the Center for Cancer Research.

\section{Author details}

'Genetic Epidemiology Branch, Division of Cancer Epidemiology and Genetics, National Cancer Institute, NIH, DHHS, Bethesda, Maryland, USA. 'Laboratory of Population Genetics, Center for Cancer Research, National Cancer Institute, NIH, DHHS, Bethesda, Maryland, USA. ${ }^{3}$ Shanxi Cancer Hospital, Taiyuan, Shanxi, 030013, PR China.

\section{Authors' contributions}

$\mathrm{NH}, \mathrm{AMG}, \mathrm{TD}$, and PRT designed, conducted, and supervised the field and clinical studies; PRT obtained funding for the project; $\mathrm{NH}$ and $\mathrm{CW}$ designed and performed the laboratory analyses; $\mathrm{NH}, \mathrm{RJC}, \mathrm{HHY}$, and MPL conducted the statistical analyses; $\mathrm{NH}$ and ML drafted the manuscript; $\mathrm{NH}, \mathrm{RJC}, \mathrm{AMG}$, PRT, and MPL conceptualized the data analyses and revised and edited the manuscript. All authors read and approved the final manuscript.

Received: 28 February 2010 Accepted: 18 October 2010 Published: 18 October 2010

\section{References}

1. Huang J, Wei W, Zhang J, Liu G, Bignell GR, Stratton MR, et al: Whole genome DNA copy number changes identified by high density oligonucleotide arrays. Hum Genomics 2004, 1:287-299.

2. Bignell GR, Huang J, Greshock J, Watt S, Butler A, West S, et al: Highresolution analysis of DNA copy number using oligonucleotide microarrays. Genome Res 2004, 14:287-295.

3. Teh MT, Blaydon D, Chaplin T, Foot NJ, Skoulakis S, Raghavan M, et al: Genomewide single nucleotide polymorphism microarray mapping in basal cell carcinomas unveils uniparental disomy as a key somatic event. Cancer Res 2005, 65:8597-8603.

4. Fitzgibbon J, Smith LL, Raghavan M, Smith ML, Debernardi S, Skoulakis S, et al: Association between acquired uniparental disomy and homozygous gene mutation in acute myeloid leukemias. Cancer Res 2005, 65:9152-9154.

5. Raghavan M, Lillington DM, Skoulakis S, Debernardi S, Chaplin T, Foot NJ, et al: Genome-wide single nucleotide polymorphism analysis reveals frequent partial uniparental disomy due to somatic recombination in acute myeloid leukemias. Cancer Res 2005, 65:375-378

6. Langdon JA, Lamont JM, Scott DK, Dyer S, Prebble E, Bown N, et al: Combined genome-wide allelotyping and copy number analysis identify frequent genetic losses without copy number reduction in medulloblastoma. Genes Chromosomes Cancer 2006, 45:47-60

7. Stark M, Hayward N: Genome-wide loss of heterozygosity and copy number analysis in melanoma using high-density single-nucleotide polymorphism arrays. Cancer Res 2007, 67:2632-2642.

8. Ross CW, Ouillette PD, Saddler CM, Shedden KA, Malek SN: Comprehensive analysis of copy number and allele status identifies multiple chromosome defects underlying follicular lymphoma pathogenesis. Clin Cancer Res 2007, 13:4777-4785.

9. Gaasenbeek M, Howarth K, Rowan AJ, Gorman PA, Jones A, Chaplin T, et al: Combined array-comparative genomic hybridization and singlenucleotide polymorphism-loss of heterozygosity analysis reveals complex changes and multiple forms of chromosomal instability in colorectal cancers. Cancer Res 2006, 66:3471-3479.

10. Andersen CL, Wiuf C, Kruhoffer M, Korsgaard M, Laurberg S, Orntoft TF: Frequent occurrence of uniparental disomy in colorectal cancer. Carcinogenesis 2007, 28:38-48

11. van PM, Middeldorp A, Tops CM, van ER, van der Klift HM, Vasen HF, et al: Genome-wide copy neutral LOH is infrequent in familial and sporadic microsatellite unstable carcinomas. Fam Cancer 2008, 7:319-330.

12. Lo KC, Bailey D, Burkhardt T, Gardina P, Turpaz Y, Cowell JK: Comprehensive analysis of loss of heterozygosity events in glioblastoma using the 100K SNP mapping arrays and comparison with copy number abnormalities defined by BAC array comparative genomic hybridization. Genes Chromosomes Cancer 2008, 47:221-237.

13. Kuga D, Mizoguchi M, Guan Y, Hata N, Yoshimoto K, Shono T, et al: Prevalence of copy-number neutral LOH in glioblastomas revealed by genomewide analysis of laser-microdissected tissues. Neuro Oncol 2008, 10:995-1003.

14. Purdie KJ, Lambert SR, Teh MT, Chaplin T, Molloy G, Raghavan M, et al: Allelic imbalances and microdeletions affecting the PTPRD gene in cutaneous squamous cell carcinomas detected using single nucleotide polymorphism microarray analysis. Genes Chromosomes Cancer 2007, 46:661-669.

15. Akagi $T$, Shih $L Y$, Kato M, Kawamata N, Yamamoto G, Sanada M, et al: Hidden abnormalities and novel classification of $\mathrm{t}(15 ; 17)$ acute promyelocytic leukemia (APL) based on genomic alterations. Blood 2009, 113:1741-1748

16. Sulong S, Moorman AV, Irving JA, Strefford JC, Konn ZJ, Case MC, et al: A comprehensive analysis of the CDKN2A gene in childhood acute lymphoblastic leukemia reveals genomic deletion, copy number neutral loss of heterozygosity, and association with specific cytogenetic subgroups. Blood 2009, 113:100-107.

17. Gorringe KL, Ramakrishna M, Williams LH, Sridhar A, Boyle SE, Bearfoot JL, et al: Are there any more ovarian tumor suppressor genes? A new perspective using ultra high-resolution copy number and loss of heterozygosity analysis. Genes Chromosomes Cancer 2009, 48:931-942.

18. Nancarrow DJ, Handoko HY, Smithers BM, Gotley DC, Drew PA, Watson DI, et al: Genome-wide copy number analysis in esophageal adenocarcinoma using high-density single-nucleotide polymorphism arrays. Cancer Res 2008, 68:4163-4172.

19. O'Keefe C, McDevitt MA, Maciejewski JP: Copy neutral loss of heterozygosity: a novel chromosomal lesion in myeloid malignancies. Blood 2010, 115:2731-2739.

20. Li JY: Epidemiology of esophageal cancer in China. Natl Cancer Inst Monogr 1982, 62:113-120

21. Qiao YL, Hou J, Yang L, He YT, Liu YY, Li LD, et al: [The trends and preventive strategies of esophageal cancer in high-risk areas of Taihang Mountains, China]. Zhongguo Yi Xue Ke Xue Yuan Xue Bao 2001, 23:10-14.

22. Hu N, Roth MJ, Emmert-Buck MR, Tang ZZ, Polymeropolous M, Wang QH, et al: Allelic loss in esophageal squamous cell carcinoma patients with and without family history of upper gastrointestinal tract cancer. Clin Cancer Res 1999, 5:3476-3482.

23. Hu N, Roth MJ, Polymeropolous M, Tang ZZ, Emmert-Buck MR, Wang QH, et al: Identification of novel regions of allelic loss from a genomewide scan of esophageal squamous-cell carcinoma in a high-risk Chinese population. Genes Chromosomes Cancer 2000, 27:217-228.

24. Huang J, Hu N, Goldstein AM, Emmert-Buck MR, Tang ZZ, Roth MJ, et al: High frequency allelic loss on chromosome 17p13.3-p11.1 in esophageal squamous cell carcinomas from a high incidence area in northern China. Carcinogenesis 2000, 21:2019-2026.

25. Hu N, Su H, Li WJ, Giffen C, Goldstein AM, Hu Y, et al: Allelotyping of esophageal squamous-cell carcinoma on chromosome 13 defines deletions related to family history. Genes Chromosomes Cancer 2005, 44:271-278.

26. Hu N, Wang C, Hu Y, Yang HH, Kong LH, Lu N, et al: Genome-wide loss of heterozygosity and copy number alteration in esophageal squamous cell carcinoma using the Affymetrix GeneChip Mapping $10 \mathrm{~K}$ array. BMC Genomics 2006, 7:299.

27. Hu N, Wang C, Ng D, Clifford R, Yang HH, Tang ZZ, et al: Genomic characterization of esophageal squamous cell carcinoma from a highrisk population in China. Cancer Res 2009, 69:5908-5917.

28. Hu N, Flaig MJ, Su H, Shou JZ, Roth MJ, Li WJ, et al: Comprehensive characterization of annexin I alterations in esophageal squamous cell carcinoma. Clin Cancer Res 2004, 10:6013-6022.

29. Li G, Hu N, Goldstein AM, Tang ZZ, Roth MJ, Wang QH, et al: Allelic loss on chromosome bands 13q11-q13 in esophageal squamous cell carcinoma. Genes Chromosomes Cancer 2001, 31:390-397.

30. Hu N, Li G, Li WJ, Wang C, Goldstein AM, Tang ZZ, et al: Infrequent mutation in the BRCA2 gene in esophageal squamous cell carcinoma. Clin Cancer Res 2002, 8:1121-1126.

31. Ng D, Hu N, Hu Y, Wang C, Giffen C, Tang ZZ, et al: Replication of a genome-wide case-control study of esophageal squamous cell carcinoma. Int J Cancer 2008, 123:1610-1615.

32. Gao Y, Hu N, Han X, Giffen C, Ding T, Goldstein A, et al: Family history of cancer and risk for esophageal and gastric cancer in Shanxi, China. BMC Cancer 2009, 9:269. 
33. Gao $Y$, Hu N, Han X, Giffen C, Ding T, Goldstein AM, et al: Jasmine tea consumption and upper gastrointestinal cancer in China. Cancer Causes Control 2009, 20:1997-2007.

34. Emmert-Buck MR, Bonner RF, Smith PD, Chuaqui RF, Zhuang Z, Goldstein SR, et al: Laser capture microdissection. Science 1996, 274:998-1001.

35. Irizarry RA, Hobbs B, Collin F, Beazer-Barclay YD, Antonellis KJ, Scherf U, et al: Exploration, normalization, and summaries of high density oligonucleotide array probe level data. Biostatistics 2003, 4:249-264.

36. Bolstad BM, Irizarry RA, Astrand M, Speed TP: A comparison of normalization methods for high density oligonucleotide array data based on variance and bias. Bioinformatics 2003, 19:185-193.

doi:10.1186/1471-2164-11-576

Cite this article as: Hu et al:: Genome wide analysis of DNA copy number neutral loss of heterozygosity (CNNLOH) and its relation to gene expression in esophageal squamous cell carcinoma. BMC Genomics 2010 11:576.

\section{Submit your next manuscript to BioMed Central} and take full advantage of:

- Convenient online submission

- Thorough peer review

- No space constraints or color figure charges

- Immediate publication on acceptance

- Inclusion in PubMed, CAS, Scopus and Google Scholar

- Research which is freely available for redistribution

Submit your manuscript at www.biomedcentral.com/submit 